\title{
Análisis estadístico de la resistencia mecánica de materiales cerámicos de mullita sometidos a diferentes ciclos de calentamiento
}

\author{
J. PASCUAL COSP, A. J. RAMírez Del VAlLE, J. ZAPATERO ARENZANA, J. C. GALIANO SERRANO \\ Dpto. Ingeniería Civil, de Materiales y Fabricación. ETSII. Universidad de Málaga. Campus de El Ejido s/n. 29013 Málaga
}

\begin{abstract}
Las aplicaciones propias de los materiales cerámicos de mullita propician que a lo largo de su vida útil se vean sometidos a importantes solicitaciones mecánicas y térmicas. Es por ello interesante establecer procedimientos que permitan predecir su comportamiento en función del estado inicial. Para este estudio se utilizó una mullita del tipo $2 \mathrm{Al}_{2} \mathrm{O}_{3}: 1 \mathrm{SiO}_{2}$ obtenida usando un método de procesado químico a partir de caolinita y residuos de aluminio. Se fabricaron varias series de dicho material para ser sometidas a distintos ciclos de cocción que proporcionasen materiales con diferente grado de sinterización. La resistencia a la fractura de dichas cerámicas se determinó mediante ensayo a flexión en cuatro puntos. La función de distribución de Weibull se usó para caracterizar estadísticamente la variación de la resistencia mecánica. A continuación se procedió al estudio de su superficie por microscopía electrónica de barrido, para establecer un mapeado de defectos que posibilitase la obtención de la distribución de tamaño de poro, orientación y factor de forma de cada probeta. Finalmente se llevó a cabo un estudio de resistencia usando la teoría de Weibull y el mapeado de defectos superficiales.
\end{abstract}

Palabras clave: mullita, Weibull, resistencia mecánica, sinterización, defectos superficiales..

\section{Statistical analysis of the mechanical resistance of mullite ceramics materials under different heating processes}

The applications of mullite based ceramics cause that along their useful life they are subjected to important mechanical and thermal solicitations. This is the reason why it is very interesting to establish procedures that allow to predict their behaviour in function of the initial condition. For this work the ceramic used was a $2 \mathrm{Al}_{2} \mathrm{O}_{3}: 1 \mathrm{SiO}_{2}$ mullite material obtained using a method of chemical processing of kaolinite and aluminium metal wastes. Mullite based ceramic materials with different sintering grade were obtained using various firing conditions. Strength of ceramics was measured in four point bending test. Weibull distribution function was used to characterize statistically the variation of the mechanical strength. A surface flaws mapping was established by scanning electron microscopy and distributions of pore-size, orientation and shape factor were obtained for each sample. A study of strength is done using Weibull's theory and the surface flaws mapping.

Keywords: mullite, Weibull, mechanical strength, sintering, surface flaws..

\section{INTRODUCCIÓN}

El amplio espectro de posibilidades de utilización de las cerámicas tecnológicas deriva de sus buenas propiedades mecánicas, termomecánicas, eléctricas, químicas, etc. De entre todas ellas, los materiales cerámicos de mullita han recibido una gran atención en las últimas décadas. Desde el punto de vista mecánico, la propiedad de la mullita más estudiada ha sido la fluencia, si bien también se han realizado trabajos sobre los mecanismos que intervienen en el proceso de fractura (1). Por otra parte partiendo de materiales compuestos mullita/ circonia se han determinado las correlaciones existentes entre la microestructura y las propiedades mecánicas bajo diferentes ciclos de cocción (2).

Sin embargo, las singularidades que presentan estos materiales, ligadas a las condiciones de procesado, pueden incidir negativamente en estas expectativas. Estos materiales presentan una gran dispersión en cuanto a su resistencia mecánica (3), debido fundamentalmente a la población de microdefectos característica de los materiales frágiles. Estos microdefectos se encuentran distribuidos estadísticamente en función de su clase, forma, orientación, posición y tamaño. Para poder analizar dichos datos es necesario realizar un análisis estadístico de los mismos.
Entre las funciones de distribución estadísticas, el modelo de Weibull (4) es el más ampliamente utilizado para analizar las medidas de resistencia y predecir el tiempo de vida útil de los componentes cerámicos. Es un modelo muy apropiado debido a su adaptabilidad a los datos experimentales y su relativa simplicidad matemática.

En los materiales cerámicos las grietas volumétricas o superficiales que actúan como concentradores de tensiones determinan la resistencia a la fractura de los mismos. La fractura del material se produce cuando se alcanza la tensión suficiente en alguna grieta para producir el crecimiento inestable de la misma. En una muestra bajo un estado de tensión uniforme, es la grieta crítica (por su tamaño, forma $u$ orientación) la que determina la resistencia del elemento. El modelo de Weibull se basa en el principio del eslabón más débil, por el cual la estructura del material se considera como una cadena cuya resistencia es controlada por el eslabón mas débil, que es equivalente a la región donde se encuentra la grieta más crítica.

La función de distribución acumulativa de fallo $\mathrm{F}(\sigma)$ de un cuerpo usando el modelo de Weibull es (5): 


$$
\mathrm{F}(\sigma)=1-\operatorname{Exp}\left[-\left(\sigma / \sigma_{0}\right)^{\mathrm{m}}\right]
$$

Tomando dos veces logaritmo, obtenemos la siguiente ecuación para la probabilidad de supervivencia $(1-\mathrm{F}(\sigma))$ :

$$
\operatorname{LnLn}[1 /(1-\mathrm{F}(\sigma))]=\operatorname{mLn}(\sigma)+\operatorname{Ln}\left(1 / \sigma_{0}\right)^{\mathrm{m}} .
$$

Esta ecuación se puede dibujar como una línea recta $\operatorname{LnLn}[1 /(1-\mathrm{F}(\sigma))]$ frente a $\mathrm{mLn}(\sigma)$ cuya pendiente es el módulo de Weibull $\mathrm{m}$ y cuya intersección en el origen es $\operatorname{Ln}\left(1 / \sigma_{0}\right)^{\mathrm{m}}$.

Los parámetros $\mathrm{m}$ y $\sigma_{0}$ se determinan gráficamente o numéricamente mediante el método de mínimos cuadrados. Para este propósito, se debe asignar una probabilidad de fallo $F_{i}$ a cada valor de $\sigma_{i}$ después de ordenar todos los valores medidos en orden ascendente de acuerdo con el siguiente estimador de probabilidad $\mathrm{F}_{\mathrm{i}}=(\mathrm{i}-0.5) / \mathrm{n}$, siendo i la posición que ocupa la resistencia a la fractura dentro de la ordenación realizada y $n$ el número de muestras ordenadas (6).

Diferentes autores $(7,8)$ han mostrado la conveniencia de usar una función de peso, $\mathrm{W}_{\mathrm{i}}$, para llevar a cabo la regresión lineal. Bergman (7) propuso el siguiente factor de peso:

$$
\mathrm{W}_{\mathrm{i}}=\left[\left(1-\mathrm{F}_{\mathrm{i}}\right) \times \ln \left(1-\mathrm{F}_{\mathrm{i}}\right)\right]^{2} \text {. }
$$

Mientras Faucher y Tyson [8] propusieron el siguiente:

$$
\mathrm{W}_{\mathrm{i}}=3.3 \mathrm{~F}_{\mathrm{i}}-27.5\left[1-\left(1-\mathrm{F}_{\mathrm{i}}\right)^{0.025}\right] \text {. }
$$

Haciendo uso de una función de peso los parámetros de la distribución de Weibull pueden ser calculados como

$$
\begin{aligned}
& \mathrm{m}=\left[\left(\sum \mathrm{W}_{\mathrm{i}}\right)\left(\sum \mathrm{W}_{\mathrm{i}} \mathrm{z}_{\mathrm{i}} \mathrm{y}_{\mathrm{i}}\right)-\left(\sum \mathrm{W}_{\mathrm{i}} \mathrm{z}_{\mathrm{i}}\right)\left(\sum \mathrm{W}_{\mathrm{i}} \mathrm{y}_{\mathrm{i}}\right)\right] /\left[\left(\sum \mathrm{W}_{\mathrm{i}}\right)\left(\sum \mathrm{W}_{\mathrm{i}} \mathrm{z}_{\mathrm{i}}^{2}\right)-\left(\sum \mathrm{W}_{\mathrm{i}} \mathrm{z}_{\mathrm{i}}\right)^{2}\right] . \\
& \quad \sigma_{0}=\exp (-\mathrm{a} / \mathrm{m})
\end{aligned}
$$

donde

$\mathrm{z}_{\mathrm{i}}=\ln \sigma_{\mathrm{i}}$

$$
\begin{aligned}
& \mathrm{y}_{\mathrm{i}}=\ln [\ln (1 /(1-\mathrm{F}))] \\
& \mathrm{a}=\left[\Sigma \mathrm{W}_{\mathrm{i}} \mathrm{y}_{\mathrm{i}}-\mathrm{m} \Sigma \mathrm{W}_{\mathrm{i}} \mathrm{z}_{\mathrm{i}}\right] / \Sigma \mathrm{W}_{\mathrm{i}}
\end{aligned}
$$

Para la aplicación del modelo de Weibull se considera el material como un medio homogéneo con una población de defectos, en un número suficiente, distribuidos aleatoriamente dentro de cada muestra. En el caso de que existan dos familias de defectos que controlen la resistencia, el gráfico de Weibull $(\ln [\ln (1 /(1-F))]$ frente a $\ln \sigma)$ presentará un comportamiento bimodal (aparecerá un mejor ajuste a dos rectas).

\section{MATERIALES Y MÉTODOS}

\subsection{Materiales.}

Para este estudio se utilizó una mullita del tipo $2 \mathrm{Al}_{2} \mathrm{O}_{3}: 1 \mathrm{SiO}_{2}$ obtenida usando el método propuesto por Pascual y colaboradores (9) mediante procesado químico a partir de caolinita de Poveda de la Sierra y residuos de aluminio.

\subsection{Métodos.}

Las muestras de mullita se obtuvieron como barras prismáticas de dimensiones $10 \times 10 \times 50 \mathrm{~mm}$ después de su compactación mediante una presión de $130 \mathrm{MPa}$ en una prensa uniaxial. A continuación fueron sinterizadas y sometidas a distintos ciclos de calentamiento-enfriamiento en un horno de cámara siguiendo diferentes programas. En los programas escogidos, la primera etapa se realizaba para el proceso de sinterización y los restantes etapas simulaban diferentes ciclos de operación. Los programas seguidos se exponen en la Tabla 1.

Las muestras de las series M1 y M2 se pulieron, reduciendo

\begin{tabular}{|c|c|c|c|c|c|c|c|}
\hline Serie & M1 & & & & & & \\
\hline Etapa & $\begin{array}{l}\text { Temperatura } \\
\text { inicial }\left({ }^{\circ} \mathrm{C}\right)\end{array}$ & \multicolumn{2}{|c|}{$\begin{array}{c}\text { Rampa calentamiento } \\
\left({ }^{\circ} \mathrm{C} / \mathrm{min}\right)\end{array}$} & $\begin{array}{l}\text { Temperatura de } \\
\text { recocido }\left({ }^{\circ} \mathrm{C}\right)\end{array}$ & $\begin{array}{l}\text { Tiempo de } \\
\text { recocido (min) }\end{array}$ & $\begin{array}{c}\text { Rampa } \\
\text { enfriamiento } \\
\left({ }^{\circ} \mathrm{C} / \mathrm{min}\right)\end{array}$ & $\begin{array}{c}\text { Temperatura final } \\
\left({ }^{\circ} \mathrm{C}\right)\end{array}$ \\
\hline 1 & 70 & \multicolumn{2}{|c|}{25} & 1570 & 300 & 50 & 70 \\
\hline 2 & 70 & \multicolumn{2}{|c|}{25} & 1570 & 300 & 50 & 70 \\
\hline Serie & M2 & & & & & & \\
\hline Etapa & $\begin{array}{l}\text { Temperatura } \\
\text { inicial }\left({ }^{\circ} \mathrm{C}\right)\end{array}$ & $\begin{array}{c}\text { Rampa } \\
\text { calentamiento } \\
\left({ }^{\circ} \mathrm{C} / \mathrm{min}\right) \\
\end{array}$ & \multicolumn{2}{|c|}{ Temperatura de recocido $\left({ }^{\circ} \mathrm{C}\right)$} & $\begin{array}{l}\text { Tiempo de } \\
\text { recocido (min) }\end{array}$ & $\begin{array}{c}\text { Rampa } \\
\text { enfriamiento } \\
\left({ }^{\circ} \mathrm{C} / \mathrm{min}\right) \\
\end{array}$ & $\begin{array}{l}\text { Temperatura final } \\
\left({ }^{\circ} \mathrm{C}\right)\end{array}$ \\
\hline 1 & 70 & 25 & \multicolumn{2}{|c|}{1570} & 300 & 50 & 800 \\
\hline 2 & 800 & 25 & \multicolumn{2}{|c|}{1570} & 360 & 50 & 70 \\
\hline Serie & M3 & & & & & & \\
\hline Etapa & $\begin{array}{c}\text { Temperatura } \\
\text { inicial }\left({ }^{\circ} \mathrm{C}\right)\end{array}$ & $\begin{array}{c}\text { Rampa } \\
\text { calentamiento } \\
\left({ }^{\circ} \mathrm{C} / \mathrm{min}\right) \\
\end{array}$ & \multicolumn{2}{|c|}{ Temperatura de recocido $\left({ }^{\circ} \mathrm{C}\right)$} & $\begin{array}{l}\text { Tiempo de } \\
\text { recocido }(\mathrm{min})\end{array}$ & $\begin{array}{c}\text { Rampa } \\
\text { enfriamiento } \\
\left({ }^{\circ} \mathrm{C} / \mathrm{min}\right)\end{array}$ & $\begin{array}{l}\text { Temperatura final } \\
\left({ }^{\circ} \mathrm{C}\right)\end{array}$ \\
\hline 1 & 70 & 25 & \multicolumn{2}{|c|}{1570} & 300 & 50 & 70 \\
\hline 3 & 70 & 25 & \multicolumn{2}{|c|}{1650} & 240 & 50 & 70 \\
\hline
\end{tabular}
el tamaño de la pasta de diamante usada de 15 a $6 \mu \mathrm{m}$ y finalmente a $3 \mu \mathrm{m}$ mediante una pulidora automática plana, obteniéndose unas dimensiones finales de 7,5 × 6,5 × 45 $\mathrm{mm}$. Las muestras de la serie M3 fueron cortadas y pulidas siguiendo el mismo procedimiento que para las anteriores series obteniéndose unas dimensiones finales de 3,5 $\times 3 \times 45$ $\mathrm{mm}$.

TABLA I. PROGRAMAS DE COCCIÓN DE LOS DIFERENTES MATERIALES DE MULLITA ENSAYADOS 
El estudio de los defectos superficiales se realizó para cada muestra mediante microscopía electrónica de barrido, usando un equipo JEOL JSM 6400, tomándose aproximadamente 200 imágenes de cada probeta a 500 aumentos. Se trabajó a una tensión de aceleración de $5 \mathrm{Kv}$ para que el volumen de interacción de los electrones con el material fuese el menor posible obteniéndose una imagen lo más superficial posible. Se analizaron las imágenes de electrones secundarios mediante un programa de análisis de imagen propio desarrollado para tal fin, que proporciona las distribuciones de tamaño de poro, orientación y factor de forma de los defectos.

El ensayo de flexión en cuatro puntos de las diferentes series de material se realizó a temperatura ambiente usando una máquina de fatiga, Instron 8500 , con una velocidad de desplazamiento de la célula de carga de $0.5 \mathrm{~mm} \mathrm{~min}^{-1}$.

\section{RESULTADOS EXPERIMENTALES Y DISCUSIÓN}

Una vez realizado el ensayo a flexión en cuatro puntos de las diferentes series de probetas se procedió al análisis estadístico de los datos dentro de cada serie. Para ello se ordenaron los valores de resistencia dentro de cada serie de menor a mayor y se asignó a cada valor de su tensión de fractura $\sigma_{i}$, una probabilidad de fallo $\mathrm{F}_{\mathrm{i}}$ haciendo uso del estimador estadístico $F_{i}=(i-0.5) / n$. Las diferentes funciones de distribución acumulativa se muestran en la Fig. 1.

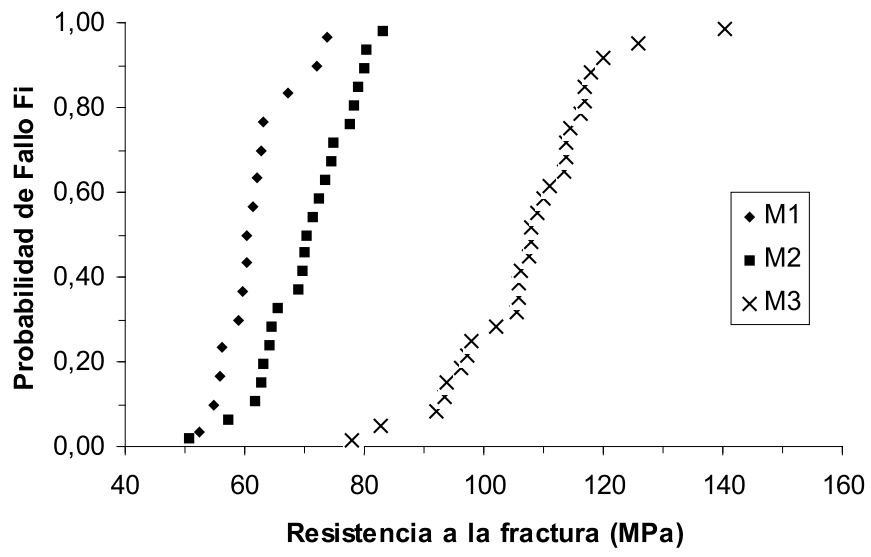

Fig. 1.- Funciones de distribución acumulativa de las diferentes series

En la Fig. 2. se representan los gráficos de Weibull de las diferentes series. Dichas distribuciones se ajustan bien a una sola recta, con lo que se puede concluir que el material bajo estudio presenta un carácter unimodal en su comportamiento

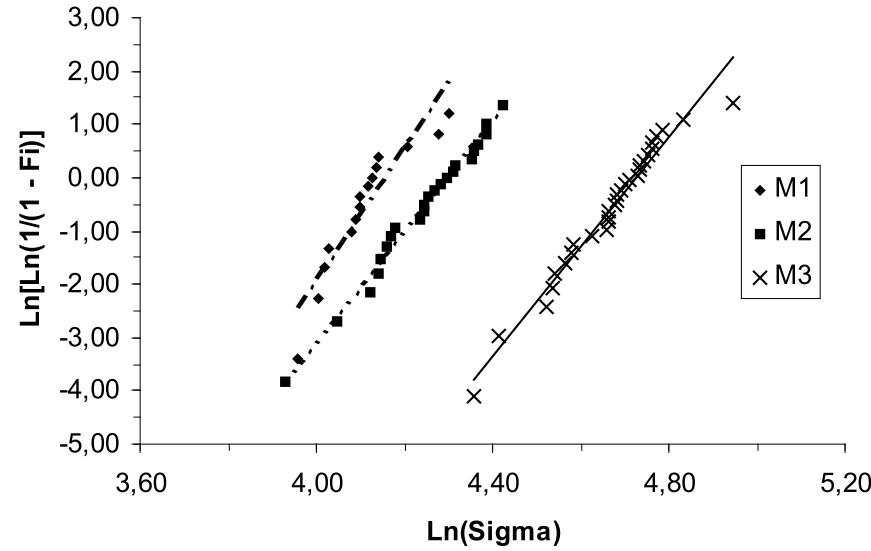

Fig. 2.- Funciones de Weibull de las diferentes series de mullita ensayadas.

resistente, siendo el fallo del material debido a una única familia de defectos. Si por el contrario los datos obtenidos se ajustasen mejor a dos rectas, esto nos indicaría que deberíamos utilizar una distribución de Weibull bimodal, donde el fallo del material vendría gobernado por la acción conjunta de las familias de defectos superficiales y volumétricos.

A continuación se procedió a la obtención de los parámetros característicos de la distribución de Weibull haciendo uso de los métodos de regresión lineal, y regresión lineal con función de peso, usando como tales las descritas en las ecuaciones [3] y [4]. La Tabla 2. muestra los valores de dichos parámetros para las diferentes series de material bajo estudio. Para cada serie se tomó como valor característico de la distribución el valor promedio de los parámetros obtenidos usando los diferentes métodos de interpolación.

Para proceder a un estudio comparativo entre las resistencias a la fractura de los materiales obtenidos para los diferentes programas de cocción, se hizo uso de la ecuación de efecto tamaño ya que las muestras de la serie M3 tenían unas dimensiones diferentes a las del resto. Asumiendo que los defectos superficiales eran los responsables del fallo, como es el caso presente, la ecuación del efecto tamaño viene dada por (10):

$$
\sigma_{1} / \sigma_{2}=\left[S_{e f, 2} / S_{e f, 1}\right]^{1 / m}
$$

La resistencia característica ajustada a una superficie efectiva de $1 \mathrm{~mm}^{2}, \sigma_{0 s^{\prime}}$ puede ser obtenida para una muestra con unas dimensiones específicas usando la ecuación del efecto tamaño (10) como

$$
\sigma_{0 \mathrm{~S}}=\sigma_{0}\left[\mathrm{~S}_{\mathrm{ef}, \mathrm{muestra}} / \mathrm{S}_{\mathrm{ef}, 0}\right]^{1 / \mathrm{m}}=\sigma_{0}\left[\mathrm{~S}_{\mathrm{ef}, \text { muestra }} / 1 \mathrm{~mm}^{2}\right]^{1 / \mathrm{m}}
$$

TABLA II. PARÁMETROS CARACTERÍSTICOS DE LA DISTRIBUCIÓN DE WEIBULL PARA LAS DIFERENTES SERIES USANDO DISTINTOS MÉTODOS DE INTERPOLACIÓN.

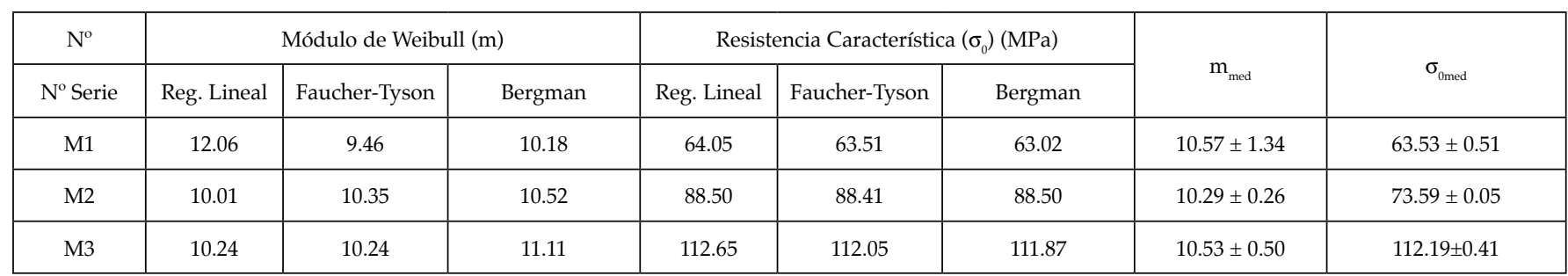


TABLA III. RESULTADOS DE SUPERFICIE EFICAZ Y RESISTENCIA CARACTERÍSTICA REDUCIDA A UNA SUPERFICIE DE $1 \mathrm{MM}^{2}$ DE LOS DISTINTOS TIPOS DE MATERIAL.

\begin{tabular}{|c|c|c|}
\hline Serie de material & $\begin{array}{c}\text { Superficie efectiva }\left(\mathrm{S}_{\text {efec }}\right) \\
\left(\mathrm{mm}^{2}\right)\end{array}$ & $\begin{array}{c}\text { Resist. Característica } \\
\left(\sigma_{05}\right)(\mathrm{MPa})\end{array}$ \\
\hline M1 & 174.56 & 103.53 \\
\hline M2 & 173.58 & 123.61 \\
\hline M3 & 79.57 & 170.00 \\
\hline
\end{tabular}

La superficie efectiva de la muestra para el ensayo a flexión en cuatro puntos viene determinada por la siguiente ecuación (11):

$$
\mathrm{S}_{\text {efectiva }}=\mathrm{hl}(1+\mathrm{km}) /(1+\mathrm{m})^{2}+\mathrm{wl}(1+\mathrm{km}) /(1+\mathrm{m}) .
$$

Donde $\mathrm{k}=\mathrm{l}_{1} / \mathrm{l}$; siendo $\mathrm{l}_{1}$ la distancia entre apoyos internos y 1 la distancia entre apoyos externos del útil de flexión; w y $\mathrm{h}$ son el ancho y el alto de la muestra, y m es el módulo de Weibull.

Los resultados para las diferentes series se muestran en la Tabla 3.

De igual forma, la resistencia media obtenida a partir de los resultados del ensayo de flexión y ajustada a una superficie efectiva de $1 \mathrm{~mm}^{2}, \sigma_{\mathrm{ms}}$, se calcula modificando la ecuación anterior de la siguiente forma:

$$
\sigma_{\mathrm{mS}}=\sigma_{\mathrm{m}}\left[\mathrm{S}_{\text {ef,muestra }} / \mathrm{S}_{\mathrm{ef}, \mathrm{m}}\right]^{1 / \mathrm{m}}=\sigma_{0}\left[\mathrm{~S}_{\mathrm{ef}, \mathrm{muestra}} / 1 \mathrm{~mm}^{2}\right]^{1 / \mathrm{m}} .
$$

La Fig. 3. corresponde a la representación de las resistencias a la fractura media y la desviación típica de las diferentes series ensayadas para una superficie efectiva de $1 \mathrm{~mm}^{2}$. Dicha gráfica permite extraer dos conclusiones, (i) la resistencia media a la fractura aumenta a medida que se aumentan el número de ciclos de cocción de la cerámica y (ii) la dispersión de los datos obtenidos también es mayor a medida que se aumenta el número de ciclos.

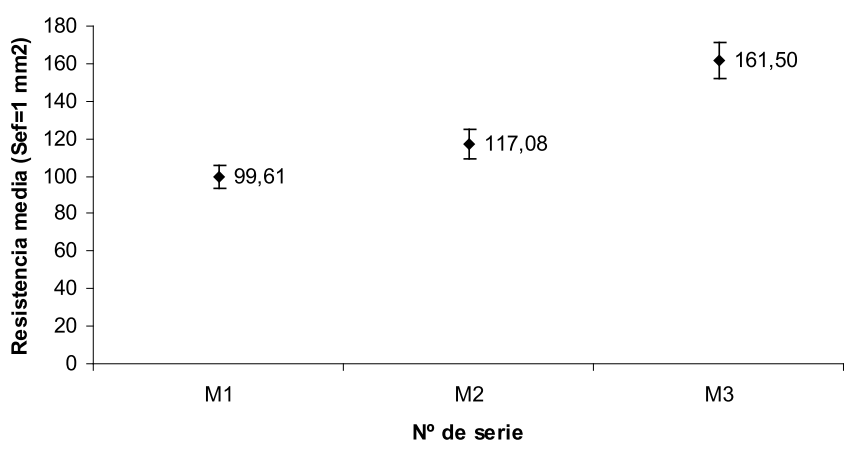

Fig. 3.- Resistencia a la fractura media de las diferentes series para una superficie efectiva de $1 \mathrm{~mm}^{2}$

Como paso siguiente del estudio se procedió a realizar el análisis de las imágenes de microscopía electrónica obtenidas para cada una de las probetas de las diferentes series mediante un programa propio desarrollado para tal fin. El análisis de microscopía cuantitativa se llevó a cabo para las series M2 y M3 que fueron las que presentaron mejores resultados en su comportamiento mecánico. El proceso seguido fue el siguiente: de cada una de las 200 imágenes obtenidas por probeta, el programa obtiene los histogramas de distribución de tamaño de poro, de orientación y factor de forma de los mismos. Para la obtención de las diferentes distribuciones se parte de la hipótesis de que la forma del poro es elíptica. A continuación el programa obtiene la distribución de tamaño de poro clasificando éstos en función de la longitud del eje mayor del poro. La distribución de orientación se realiza agrupando los poros en intervalos en función del ángulo que forme el eje mayor del mismo con la horizontal. Por último la distribución de factor de forma se calcula clasificándolos en función del cociente entre el eje mayor y menor de la elipse a la cual se asimila. Así pues éste es un parámetro que nos aporta información sobre la esfericidad del poro pues tendrá un valor unidad si el poro es esférico y será tanto mayor cuanto más se aleje el poro de dicha forma. Una vez determinadas todas las distribuciones, se obtiene la distribución media para los diferentes parámetros de todas las imágenes y se localiza la imagen cuyas funciones de distribución más se aproximen a las anteriores. Con esto se dispone de una imagen y unas distribuciones representativas de cada una de las probetas de las diferentes series. Finalmente se escoge como elemento característico de toda la serie la imagen cuyas distribuciones se aproximen más a las medias calculadas a partir de las obtenidas para cada probeta. La porosidad media de ambas series se recoge en la Tabla 4. Las imágenes representativas de las series M2 y M3 y sus histogramas correspondientes de distribuciones de tamaño de poro y factor de forma de los mismos se muestran en la Fig. 4. y Fig. 5. respectivamente. Del análisis de dichos datos se extraen las siguientes conclusiones:
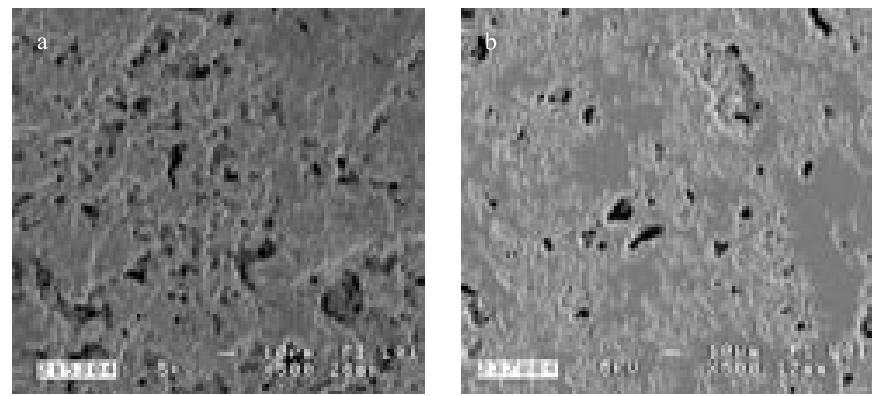

Fig. 4.- Imagen representativa de cada una de las series: (a) M2, (b) M3

- Se puede observar como se produce una disminución de la porosidad al aumentar el número de ciclos de calentamiento y como la forma de la distribución de tamaño de poro cambia al pasar de la serie M2 a la M3. Dicha disminución se debe principalmente a la desaparición de muchos poros de pequeño tamaño (0-5 micras de longitud de eje mayor del poro) como consecuencia del proceso de sinterización que hace posible la coalescencia entre los granos que rodean a dichos poros de pequeño tamaño.

- Para la distribución del factor de forma del poro por el contrario ambas series muestran distribuciones con la misma forma, pero se produce una disminución en el número de poros dentro de cada intervalo al pasar de la serie M2 a la M3. Este fenómeno es más acusado en intervalos con valores intermedios [(3-4), (4-5), (5-6)] mientras que en los valores extremos solo hay una pequeña reducción.

Esta importante disminución, induce a pensar que los 


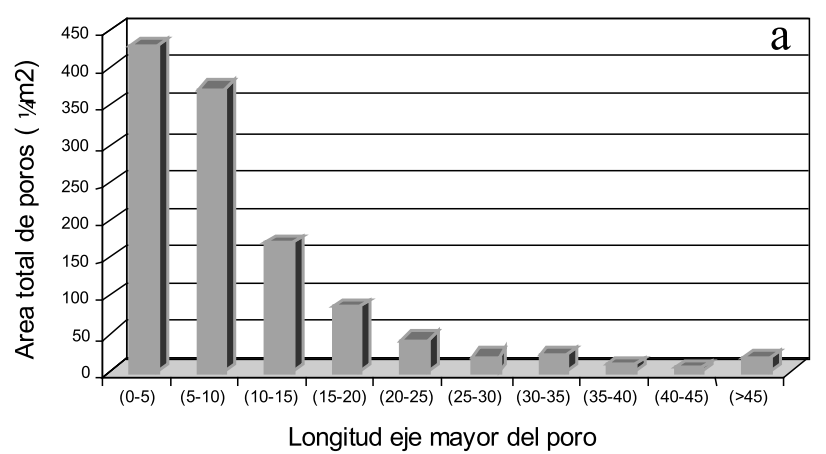

(m)

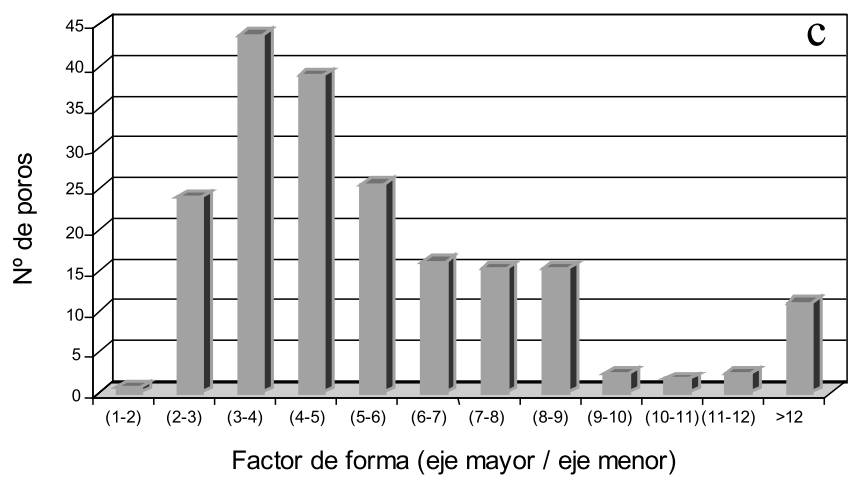

TABLA IV. POROSIDAD MEDIA DE LAS DIFERENTES SERIES DE MATERIAL

\begin{tabular}{|c|c|}
\hline Serie de material & $\begin{array}{c}\text { Porosidad media } \\
\text { (\% Área ocupada por los poros) }\end{array}$ \\
\hline M2 & 6,7363 \\
\hline M3 & 6,2236 \\
\hline
\end{tabular}

poros con dichos factores de forma que desaparecen son los causantes en gran medida de la disminución del área ocupada por los mismos en la distribución de tamaño. Así pues, el efecto que producen los sucesivos ciclos de calentamiento en la porosidad superficial observada es la desaparición de una alta cantidad de poros de pequeño tamaño y que no presentan factores de forma excesivamente altos.

El aumento de la resistencia mecánica del material al someterlo a un mayor número de ciclos de calentamiento podría ser explicada entonces como consecuencia de la acción conjunta de dos factores. Por una parte el aumento de la resistencia debido a la desaparición de la porosidad de pequeño tamaño, que hace que se disponga de un material más másico, y por otra parte al más que probable aumento de la tenacidad a la fractura que sufre dicho material al someterlo a un número mayor de ciclos.

\section{CONCLUSIONES}

De lo expuesto en el apartado anterior, se puede concluir que los materiales de mullita de estequiometría 2:1 sometidos a diferentes ciclos de calentamiento, presentan un comportamiento resistente que puede ser representado mediante el modelo probabilístico de Weibull. Además, las representaciones logarítmicas de dichas distribuciones indican

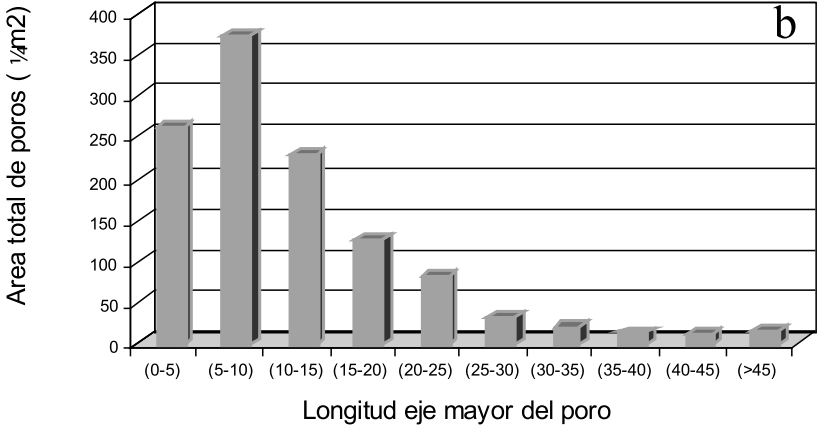

(m)

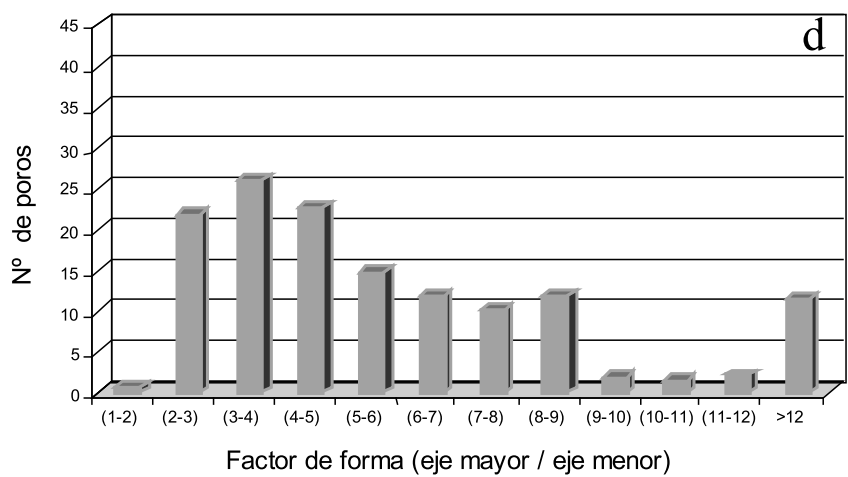

que estas cerámicas presentan un comportamiento unimodal, ya que no existen cambios de pendiente en las mismas, por lo cual los ciclos de calentamiento no influyen en la familia de defectos responsable de la fractura del material. Por otra parte, se comprueba como el modelo de efecto tamaño aplicado a superficies efectivas es adecuado para establecer relaciones entre las propiedades mecánicas de este tipo de material. Finalmente, se constata que los resultados obtenidos mediante el análisis estadístico y aquellos provenientes del estudio de microscopía cuantitativa están íntimamente relacionados.

El estudio realizado en base a un modelo probabilístico basado en un criterio global de fallo presenta un buen ajuste con los resultados de resistencia a la fractura. La utilización de modelos de fallo local usando los datos de microscopía cuantitativa nos llevaría al establecimiento de un método de ensayo que permitiría predecir la probabilidad de fallo del material para unas condiciones de servicio determinadas, y suministraría información acerca de la influencia de las condiciones de procesado en el comportamiento resistente del material.

\section{AGRADECIMIENTOS}

Los autores de este trabajo agradecen la financiación aportada por la CICYT a través del proyecto MAT99-1017C03-03.

\section{BIBLIOGRAFÍA}

1. M. Jiménez-Melendo, A. Domínguez-Rodríguez, A. Bravo-Leon. “ 1. C. Baudín, "Fracture mechanisms in a stoichiometric $3 \mathrm{Al}_{2} \mathrm{O}_{3}: 2 \mathrm{SiO}_{2}$ mullite", J. Mater. Sci. 32, 2077-2086 (1997). 
2. T. Koyama, S. Hayashi, A. Yasumori, K. Okada, M. Schmucher, H. Schneider, "Microestructure and Mechical Properties of Mullite/Zirconia Composites Prepared from Alumina and Zircon under Various Firing Conditions", J. Eur. Ceram. Soc., 16, 231-237 (1997).

3. C. A. Jhonson, W. T. Tucker, "Advanced statistical concepts of fracture in brittle materials", pp 709-716 en Engineered materials handbook, Vol 4. ASM International, (1991)

4. W. Weibull, "A statistical function of wide applicability.", J. Appl. Mech., 18, 293-297 (1951)

5. K. Trustrum, A. S. Jayatilaka, "On estimating the Weibull modulus for a brittle materials.", J. Mater. Sci., 14, 1080-1084 (1979).

6. A. D. Papargyris, "Estimator Type and Population Size for Estimating the Weibull Modulus in ceramics", J. Eur. Ceram. Soc., 18, 451-455 (1998).

7. B. Bergman. "Estimation of Weibull parameters using a weight function". J. Mater. Sci. Lett., 5, 611-614 (1986).
8. B. Faucher, R. Tyson. "On the determination of Weibull parameters". J. Mater. Sci. Lett., 7, 1199-1203 (1988).

9. J. Pascual, J. Zapatero, M. C. Jiménez de Haro, I. Varona, A. Justo, J. L. Pérez Rodríguez, P. J. Sánchez Soto. "Porous mullite and mullite-based composites by chemical processing of kaolinite and aluminium metal wastes", J. Mater. Chem., 10, 1409-1414 (2000).

10. M A. Madjoubi, C. Bousbaa, M Hamidouche y N. Bouaoadja. “Weibull Statistical Analysis of the Mechanical Strength of a Glass Eroded by Sand Blasting", J. Eur. Ceram. Soc., 19, 2957-2962, (1999).

11. H. Fischer, W. Rentzsch, R. Marx. " A modified size effect model for brittle non-metallic materials”. Eng. Fract. Mech., 69,781-791 (2002).

Recibido: 07.10 .04

Aceptado: 13.06 .05 